\title{
Interações familiares de crianças com necessidades educacionais especiais: revisão da literatura nacional
}

\author{
Paula Cruz Goitein \\ Fabiana Cia
}

\begin{abstract}
Resumo
Este trabalho teve como objetivo revisar a produção científica na literatura nacional indexada na área de Psicologia, entre 1999 e 2008 , de estudos empíricos que descreveram diferentes interações entre as crianças com NEE e seus familiares e as implicações para o desenvolvimento das mesmas, sob diferentes perspectivas teóricas. Foi realizada uma busca sistemática em três bases de dados bibliográficos (SCIELO, LILACS e Periódicos Capes), utilizando as palavras-chave: famílias de crianças com deficiência, necessidades educacionais especiais, família, relações familiares, irmãos e relações fraternais. Foram identificados 17 artigos que foram analisados considerando diferentes aspectos (periódicos em que foram publicados, variáveis relacionadas, técnicas de coleta de dados utilizadas e principais resultados obtidos). Constatou-se a carência de estudos sobre o tema em questão.
\end{abstract}

Palavras-chave: Interações familiares, necessidades educacionais especiais, deficiência.

\section{Familiar interactions of children with special educational needs: National Brazilian literature revision}

\begin{abstract}
The purpose of this study was to get a review about the scientific production shown by the Brazilian indexed literature at the psychology area for the period between 1999 and 2008. It was based on empiric studies, which described distinct interactions between children with special educational needs and their relatives, as well as the implications for their development, seen by different theoretical perspectives. A systematic search has been done using data registered in three bibliographic bases (SCIELO, LILACS and Capes periodic reviews). The key words used were: families with deficient children, special education needs, family, familiar relations, brothers, fraternal relations. A number of 17 articles was found. They were analyzed considering different aspects (periodic reviews in which they were published, related variables, collecting techniques and main results obtained. It could be seen that the theme has been scarcely studied.
\end{abstract}

Keywords: Family relations, special educational needs, deficiency.

\section{Interacciones familiares de niños con necesidades educacionales especiales: revisión de la literatura nacional}

\section{Resumen}

Este trabajo tuvo el objetivo de revisar la producción científica en la literatura nacional indexada en el área de Psicología, entre 1999 y 2008 , de estudios empíricos que describen diferentes interacciones entre niños con NEE y sus familiares, además de las consecuencias para el desarrollo de los mismos, bajo diferentes perspectivas teóricas. Se realizó una busca sistemática en tres bases de datos bibliográficos (SCIELO, LILACS e Periódicos Capes), utilizando las palabras clave: familias de niños con deficiencia, necesidades educacionales especiales, familia, relaciones familiares, hermanos y relaciones fraternales. Se identificaron 17 artículos que se analizaron considerando diferentes aspectos (periódicos en que se publicaron, variables relacionadas, técnicas de recopilación de datos utilizadas y principales resultados obtenidos). Se constató la escasez de estudios sobre el tema.

Palabras Clave: Relaciones familiares, necessidades educacionais especiais, deficiência. 


\section{Introdução}

A família tem um papel importante no desenvolvimento, sendo a base para o aprendizado de valores sociais (Del Prette, Martini, \& Del Prette, 2005; Dessen \& Silva, 2004), além de moldar as características psicológicas do indivíduo e permitir a sobrevivência da criança. Trata-se de um contexto de socialização especialmente relevante para a criança, já que, durante muitos anos, é o principal ambiente no qual ela cresce (Gomide, 2003). A família transmite valores, atitudes, cultura, conhecimento e habilidades para a vida, com base na maneira como oferece suporte emocional, social e financeiro para as crianças. Quando há uma criança com necessidades educacionais especiais (NEE ${ }^{1}$ ), o papel dos membros familiares torna-se ainda mais importante, pois a criança necessita de um maior envolvimento em seus cuidados e em sua estimulação (Yaegashi, Miranda, \& Komagrone, 2001).

Diante do nascimento de uma criança com deficiência ${ }^{2}$, há um sentimento inicial de medo e luto pela perda do filho perfeito. No geral, ambos os pais se frustram e se sentem responsáveis pela condição da criança, sendo que as mães estão mais propensas a ter depressão, por ter uma maior responsabilidade pelos cuidados e pela educação dos filhos com deficiência, quando comparadas com os pais. Muitas vezes, são elas que abdicam do trabalho remunerado e da carreira profissional para se dedicar integralmente ao filho (Paniagua, 2004; Petean \& Suguihura, 2005).

Nas pesquisas realizadas por Glidden e Floyd (1997) e Negrin e Cristante (1996), concluiu-se que as mães de crianças com deficiência apresentam maior tendência à depressão do que os pais, o que sugere que estes não vivenciam depressão ou, quando vivenciam, apresentam uma forma mais branda de depressão do que as mães. Provavelmente, isso se deve ao fato de a mãe ser a principal responsável pelos cuidados com o filho e por passar mais tempo em sua companhia. Além disso, a mãe de uma criança com deficiência pode passar a ter, quase que exclusivamente, como única função, os muitos cuidados com o filho, o que Ihe tomará muito tempo e implicará na ausência de outras atividades que poderiam servir como um auxílio para a superação das dificuldades encontradas.

Sabe-se que, no nascimento de uma criança com NEE, a família passa por diferentes fases de enfrentamento: (a) fase de choque - que ocorre ao receber a notícia de que a criança tem uma deficiência; (b) fase de negação - em que muitas famílias, após passado um tempo do recebimento da notícia, ignoram o problema ou questionam a veracidade do diagnóstico; (c) fase de reação - em que a família vive uma série de sentimentos (como irritação, culpa e depressão), os quais deve expressar, pois esta fase faz parte dos primeiros

1 Serão consideradas as crianças com necessidades educacionais especiais, com deficiência, transtornos globais de desenvolvimento ou altas habilidades/superdotação, estando de acordo com as diretrizes da Secretaria de Educação Especial do Ministério da Educação (Brasil, 2007).

20 termo deficiência será adotado para ser referir apenas às pessoas com deficiência física, auditiva, visual ou intelectual. passos de uma adaptação; e (d) fase de adaptação - em que os pais se sentem mais calmos, têm uma visão mais prática e realista da situação e procuram se organizar e se orientar para poderem ajudar os filhos. Esses sentimentos que os pais vivenciam são gerados e reforçados pelos preconceitos que a sociedade e nossa cultura impõem, uma vez que a deficiência, até os dias de hoje, é vista por muitos como um castigo, em que os pais são os principais culpados, assim eles acabam por se envergonhar com essa situação (Paniagua, 2004).

Diante disso, as famílias de criança com NEE têm maior probabilidade de vivenciarem situações estressantes, que estão relacionadas ao menor apoio social (Matsukura, Marturano, Oishi, \& Borasche, 2007). Além disso, Petean e Suguihura (2005) mostraram que existe uma sobrecarga materna diante dos cuidados da criança, pois o pai não demonstra grande envolvimento com ela, tendo o trabalho como um instrumento de fuga.

Com base nos estudos supracitados, somados à escassez de serviços públicos de saúde e educação e considerando as dificuldades de uma família lidar com as demandas de ter um filho com NEE, acredita-se que, muitas vezes, os pais, assim como os demais familiares, têm que se adaptar às necessidades e particularidades da criança, o que pode acarretar problemas no desenvolvimento tanto dos filhos com NEE, quanto dos filhos que não as têm quando os pais não conseguem ter um processo de adaptação satisfatório. Além disso, esses pais podem abdicar da realização de atividades pessoais por conta das demandas que um filho com NEE pode trazer.

Este trabalho teve como objetivo revisar a produção científica na literatura nacional indexada na área de Psicologia, entre 1999 e 2008, de estudos empíricos que descreveram diferentes interações entre as crianças com NEE e seus familiares e as implicações para o desenvolvimento das mesmas, sob diferentes perspectivas teóricas.

\section{Método}

Inicialmente, foi feita a busca por artigos nacionais a respeito da temática em revistas indexadas na base de dados LILACS, Scielo e Periódicos Capes, que disponibilizavam os artigos online. Para tanto, foram utilizados como descritores os termos: famílias de crianças com deficiência, necessidades educacionais especiais, família, relações familiares e irmãos. Essas palavras foram inseridas uma por vez e os artigos foram obtidos primeiramente na íntegra e depois selecionados os que estavam relacionados com a temática. Foram excluídos resumos de capítulos de livro ou livros relacionados a estes termos-chave. Para selecionar os artigos, procurou-se saber se os textos discorriam a respeito da família de crianças com necessidades educacionais especiais, ou a respeito de um dos integrantes dessa família, ou mesmo a respeito de um fator que poderia influenciar na relação familiar. Para delimitar a revisão, foram priorizadas apenas 
Tabela 1. Porcentagem de artigos em cada periódico

\begin{tabular}{|l|c|c|}
\hline Periódicos & (\%) & N \\
\hline Estudos de Psicologia (Campinas) & 23,5 & 4 \\
\hline Psicologia: Reflexão e Crítica & 17,6 & 3 \\
\hline Psicologia: Teoria e Pesquisa & 11,7 & 2 \\
\hline Interação em Psicologia & 11,7 & 2 \\
\hline Psicologia: Ciência e Profissão & 11,7 & 2 \\
\hline Paideia: caderno de Psicologia e Educação & 5,8 & 1 \\
\hline Psicologia em Estudo & 5,8 & 1 \\
\hline Psicologia em Revista & 5,8 & 1 \\
\hline Psicologia Escolar e Educacional & 5,8 & 1 \\
\hline & 100,0 & 17 \\
\hline
\end{tabular}

Tabela 2. Principais técnicas de coleta de dados utilizadas nos artigos

\begin{tabular}{|l|c|c|}
\hline Variáveis & $\mathbf{( \% )}$ & $\mathbf{N}$ \\
\hline Revisão de Literatura & 58,8 & 10 \\
\hline Entrevista & 35,3 & 6 \\
\hline Entrevista, aplicação de escalas e observação & 5,8 & 1 \\
\hline & $\mathbf{1 0 0 , 0}$ & $\mathbf{1 7}$ \\
\hline
\end{tabular}

revistas da área de Psicologia. Do total, foram encontrados 17 artigos nacionais, que foram obtidos na íntegra.

Com os 17 artigos nacionais selecionados, foram realizadas análises, utilizando tabelas, considerando os periódicos em que foram publicados os artigos (nome das revistas), as variáveis relacionadas (quais foram as principais variáveis investigadas em cada estudo), as técnicas de coleta de dados utilizadas e os principais resultados obtidos nos artigos. Diante disso, foi feita uma análise na qual foram comparados os dados encontrados na fundamentação teórica e os dados encontrados na revisão de literatura, que são baseados em compilação de fatos observados.

\section{Resultados e Discussão}

Os resultados e a discussão abaixo serão apresentados de forma a apontar os principais aspectos contidos nos artigos selecionados. Para tanto, será feita a seguinte divisão: (a) periódicos em que foram publicados; (b) técnicas de coleta de dados utilizadas; (c) variáveis relacionadas; e (d) principais resultados obtidos.

\section{Periódicos em que foram publicados os artigos}

A Tabela 1 apresenta a porcentagem de artigos publicados em cada periódico.
O manuscrito que mais publicou artigos nessa temática foi o Estudos de Psicologia (Canho, Neme, \& Yamado, 2006; Domingues, Motti, \& Palamin, 2008; Gomes \& Bosa, 2004; Höher \& Wagner, 2006).

\section{Técnicas de coleta de dados utilizadas}

A Tabela 2 mostra as principais técnicas de coleta de dados utilizadas nos artigos selecionados.

Pode-se constatar que a técnica de coleta de dados mais utilizada foi a de revisão de literatura (Brito \& Dessen, 1999; Fávero \& Santos, 2005; Fiamenghi \& Messa, 2007; Góes, 2006; Henn, Piccinini, \& Garcia, 2008; Nunes, Silva \& Aiello, 2008; Schmihth \& Bosa, 2003; Silva \& Dessen, 2001, 2002; Silva \& Fleith, 2008). Segundo Cozby (2006), a revisão de literatura é importante para verificar quais foram os resultados das outras pesquisas a respeito do assunto pesquisado. Mesmo em casos de pesquisas experimentais, a revisão de literatura sempre deve ser feita para ter conhecimento dos avanços e lacunas existentes (Filho \& Santos, 2001).

\section{Variáveis relacionadas}

As variáveis relacionadas em cada artigo encontramse na Tabela 3.

Como mostra a Tabela 3, as variáveis mais presentes, dentre o conjunto de artigos revisados, foram a deficiência 
Tabela 3. Porcentagem de variáveis relacionadas em cada artigo

\begin{tabular}{|l|c|c|}
\hline Variáveis & (\%) & N \\
\hline Deficiência intelectual & 5,8 & 1 \\
\hline 1- Interação entre irmãos X Deficiência intelectual & 11,7 & 2 \\
\hline 2- Impacto da família X Síndrome de Down & 17,6 & 3 \\
\hline 3- Importância da família X Deficiência intelectual & & 1 \\
\hline Deficiência auditiva & 5,8 & 1 \\
\hline 1- Brincar entre mães e filhos X Habilidades sociais X Deficiência auditiva & 5,8 & 1 \\
\hline 2- Importância da família X Deficiência auditiva & 5,8 & 1 \\
\hline 3- Vivência de mães de crianças com deficiência auditiva X Sala de espera & 5,8 & 1 \\
\hline 4- Vivência do pai X Deficiência auditiva & & 1 \\
\hline Deficiências & 5,8 & 1 \\
\hline 1- Diagnóstico X Orientação X Pais & 5,8 & 1 \\
\hline 2- Relações familiares X Deficiência & 5,8 & 17 \\
\hline 3- Importância do pai X irmão X Deficiência & 100,0 & 1,7 \\
\hline Autismo & 2 \\
\hline 1- Autismo infantil X Estresse familiar & 5,8 & 1 \\
\hline 2- Impacto da família X Autismo & & \\
\hline Superdotação & 5,8 & 1 \\
\hline 1- Importância da família X Desenvolvimento do Superdotado & \\
\hline
\end{tabular}

intelectual (Góes, 2006; Henn e cols., 2008; Nunes e cols., 2008; Silva \& Dessen, 2001, 2002; Souza \& Boemer, 2003) e a deficiência auditiva (Brito \& Dessen, 1999; Canho e cols., 2006; Domingues \& cols., 2008; Ribeiro, Yamada, \& Tavano, 2007) e sua relação com aspectos familiares.

\section{Principais resultados obtidos nos artigos}

Inicialmente, nota-se que existem poucos estudos que abordaram pesquisas sobre a família de crianças com NEE. De fato, no Brasil, ainda são raras as pesquisas que tenham participantes com NEE, porque muitos deles não estão incluídos na escola ou na sociedade. Além disso, não se tem uma estimativa precisa do número de pessoas com NEE no nosso país que são usuárias de serviços de educação e de saúde, dificultando ainda mais o acesso a elas, pois sabe-se que grande parte dessa população está à margem da escola, seja especial ou regular (Mendes, 2006; Tanaka \& Manzine, 2005). Por mais que os dados do INEP apontem para as estatísticas de crianças nas salas de aula, ainda não se tem com precisão um número de crianças com NEE porque muitas não têm diagnóstico, ou mesmo nem vão a escola, ficando sob cuidados das famílias. Tais fatores podem dificultar pesquisas com famílias de crianças com NEE.

Também são raros os estudos a respeito da repercussão de um filho superdotado na família. Na revisão de literatura, foi encontrado apenas um texto que se referia a este tema. A superdotação, no Brasil, é difícil de ser identificada, isso porque não se tem um preparo de profissionais (como professores e psicólogos) e existem poucos centros especializados na área. Alguns mitos relacionados à superdotação podem impedir ainda mais a identificação e o diagnóstico preciso, como, por exemplo: as crianças superdotadas são de famílias com melhor poder aquisitivo, têm bom desempenho em todas as matérias da escola e são disciplinadas e dedicadas (Alencar, 2007; Metrau \& Reis, 2007).

Vários estudos (Brito \& Dessen, 1999; Canho e cols., 2006; Fávero \& Santos, 2005; Fiamenghi \& Messa, 2007; Góes, 2006; Henn e cols., 2008; Nunes e cols., 2008; Schmidt \& Bosa, 2003; Silva \& Dessen, 2001, 2002; Souza \& Boemer, 2003) discutiram a repercussão do nascimento de uma criança com deficiência ou transtorno global no desenvolvimento na família. O nascimento de uma criança gera a necessidade de adaptação da família, pois é uma fase de transição. Por exemplo, os pais necessitam reajustar a rotina diária da casa (de acordo com as necessidades do bebê) e muda a dinâmica de relacionamento do casal que precisa passar a discutir, além da relação entre eles, o relacionamento entre pais e filhos e a divisão de tarefas (Bee, 2008; Palácios, 2004).

Quando a criança nasce com alguma deficiência ou com transtorno global do desenvolvimento, há um aumento na necessidade do grau de adaptação. Isso porque, para além das mudanças que qualquer família passa ao nascer um filho, essa família, muitas vezes, precisa buscar maior apoio médico, informações sobre seu filho e os aspectos 
desenvolvimentais e vivenciar mais frequentemente sentimentos de insegurança, culpa, tristeza, ansiedade, preocupação, depressão, medo e vergonha (Paniagua, 2004). Esses sentimentos são condições de enfrentamento que os pais encontram diante da situação e o estresse parental é um fator observado com muita frequência (Matsukura e cols., 2007).

Ao longo do desenvolvimento da criança com deficiência ou transtorno global do desenvolvimento, principalmente nas fases de transição (ingresso na pré-escola, no ensino fundamental, adolescência e fase adulta), os pais tendem a vivenciar sentimentos de insegurança e incertezas. Na educação infantil, muitos pais temem que seus filhos não sejam bem cuidados, sofram preconceito, etc. No ensino fundamental, as principais dúvidas se referem à escolha da escola e se a criança deve ou não ingressar em serviços de apoio. Quando chega a adolescência e a fase adulta, os pais passam a temer mais o futuro do filho em relação à independência financeira, escolarização e relacionamentos amorosos (Paniagua, 2004). No geral, os pais se preocupam mais com a independência dos filhos e com quem cuidará deles quando não puderem mais ter o auxílio dos pais (Dallabrida, 2007; Falkenbach, Drexsler, \& Werle, 2008; Pentean \& Suguihura, 2005; Silva \& Dessen, 2007; Souza \& Boemer, 2003). Nessas fases, as famílias têm maior probabilidade de passar por crises (Brito \& Dessen, 1999; Canho e cols., 2006; Fiamenghi \& Messa, 2007; Góes, 2006; Henn e cols., 2008; Pentean \& Suguihura, 2005; Silva \& Dessen, 2001, 2007).

Apesar das diversas fases de adaptação pelas quais os pais passam, quando têm um filho com NEE, isso não significa que a família terá problemas recorrentes por conta da criança, já que muitas chegam a ter um grau de satisfação com a vida e com a sua rotina igual às demais famílias de crianças com desenvolvimento típico (Paniagua, 2004).

Diversos pesquisadores apontam que os pais devem ter uma participação ativa no trabalho de intervenção precoce, uma vez que são responsáveis pela solução de problemas diários que surgem durante as várias etapas de desenvolvimento de seus filhos (Erickson \& Kurz-Riemer, 1999; Guralnick, 1998; William \& Aiello, 2004).

Os grupos de intervenção são importantes também para oferecer apoio social aos seus membros. Diferentes autores trabalharam essa questão. A família necessita de um suporte social para manutenção do equilíbrio (Dysson, 1997; Falkenbach e cols., 2008), pois existe uma relação direta entre a quantidade/satisfação com o suporte social, o nível de estresse parental e a satisfação com a família (Floyd \& Philippe, 1993; Paniagua, 2004). No entanto, muitas vezes esse suporte é escasso ou mesmo as pessoas que compõem o círculo familiar não dão apoio à família da criança com NEE por preconceito, falta de informação ou recusa de ter uma criança deficiente na família (Marchesi, 2004; Souza \& Boemer, 2003).

Como suporte social, há grupos de apoio formados por famílias que apresentam problemas semelhantes, como mostram alguns autores (Fávero \& Santos, 2005; Fiamenghi
\& Messa, 2007; Ribeiro e cols., 2007). Tais grupos são importantes, pois muitas famílias isolam-se socialmente devido à total dedicação ao cuidado com o filho com NEE (Brito \& Dessen, 1999; Fávero \& Santos, 2005; Schmidt \& Bosa, 2003; Silva \& Dessen, 2001, 2002), o que traz uma sobrecarga diante desses cuidados.

No entanto, deve-se considerar o número escasso de grupos de apoio que são oferecidos para a sociedade. Normalmente, a iniciativa da criação de tais grupos vem de instituições filantrópicas, de organizações não governamentais ou mesmo da comunidade. Além disso, não existe uma sistematização desses grupos, ou seja, não se avalia a sua efetividade, os aspectos a serem trabalhados com os pais e a necessidade das crianças. Nota-se a necessidade de políticas públicas para aumentar esse apoio aos pais. Estudos isolados de intervenção, realizados normalmente por acadêmicos, têm mostrado a efetividade dos grupos de apoio (Mendes, Almeida, \& Williams, 2004).

Apesar da importância do ingresso em programas de intervenção e grupos de apoio, a família pode demorar mais para se adaptar ou mesmo para buscar tratamentos diferenciados aos filhos com NEE, porque, muitas vezes, o diagnóstico da criança é transmitido de uma forma errada. Para Höher e Wagner (2006), pode-se constatar que profissionais da saúde não estão preparados para transmitir o diagnóstico para a família. No geral, não há preparo dos profissionais para orientar e informar a família a respeito das condições da criança (Mendes, Nunes, \& Ferreira, 2002) e muitos apontam a mãe como culpada pelo nascimento da criança deficiente, em decorrência da falta de cuidados na gestação.

Devido a esse fato, muitas famílias não buscam um tratamento precoce e não aderem a este, pois ocorre a perda de uma relação de confiança entre a família e o médico (Marchesi, 2004). Fiamenghi e Messa (2007) mostram que a adesão a tratamentos precoces traz uma melhor adaptação da família à situação do nascimento da criança com deficiência. Outros estudos (Fiamenghi \& Messa, 2007; Silva \& Dessen, 2001) demonstram que quanto mais informações a família tiver sobre as NEE, menos tensa ela fica. Para Williams e Aiello (2004), as intervenções precoces devem empoderar as famílias, ou seja, prover a família de informações e suporte para que, ao se deparar com algum problema ao longo do desenvolvimento da criança, consiga solucioná-lo. Assim, o profissional deve instrumentalizar a família, aumentando seu repertório de enfrentamento a eventos estressores e ampliando a rede de apoio já existente (Araújo, 2004; Williams \& Aiello, 2004).

Os estudos a respeito do envolvimento do pai no desenvolvimento da criança com NEE são escassos (Brito \& Dessen, 1999; Canho e cols., 2006; Henn e cols. 2008; Nunes e cols., 2008; Silva \& Dessen, 2001). O pai, conforme Canho e cols. (2006), mostra-se participante no processo de desenvolvimento de seus filhos com NEE. O papel do pai, de acordo com Nunes e cols. (2008) e Cia, Williams e Aiello (2005) vai além de provedor e mantenedor, sendo importante também para todas as áreas do desenvolvimento. Apesar 
disso, os homens ainda realizam poucas atividades, quando comparados as suas esposas (Silva, 2000). Em parte, porque muitos pais encontram no trabalho uma fuga contra o estresse familiar (Canho e cols., 2006), que é verificado, muitas vezes, nos problemas financeiros existentes devido às NEE do filho (Schmidt \& Bosa, 2003) e, outras vezes, porque alegam esse papel como sendo uma atribuição feminina.

Outro assunto que foi pouco abordado é a relação entre o irmão com desenvolvimento típico e o irmão com NEE, assim como as implicações e benefícios dessa relação. Além disso, existem poucos estudos sobre a relação dos pais com cada um desses irmãos. Conforme alguns estudos (Fiamenghi \& Messa, 2007; Gomes \& Bosa, 2004; Henn e cols., 2008; Nunes \& Aiello, 2008; Nunes e cols., 2008; Silva \& Fleith, 2008), a relação fraterna em que há um irmão com NEE e outro com desenvolvimento típico é conflitiva, mas, além dos sentimentos negativos, há também os positivos. Os irmãos com desenvolvimento típico atentam-se a conflitos familiares e assumem mais responsabilidades diante disso. Esses irmãos, segundo Gomes e Bosa (2004), apresentam um grau de satisfação na relação com os pais menor do que se comparados a irmãos de crianças com desenvolvimento típico, ou seja, o tratamento dado pelos pais a eles difere em relação ao dado para o irmão com NEE (Chacon, 2007; Nunes e cols., 2008). Contrapondo-se aos autores citados anteriormente, Nunes e Aiello (2008) afirmam que os irmãos com desenvolvimento típico não apresentam ações negativas com relação ao irmão com NEE, mostrando que os irmãos, principalmente os mais novos, ajudam os irmãos com NEE que são mais velhos, porém argumentam que ainda não foram dimensionados os problemas de irmãos de pessoas com NEE por falta de mais estudos a respeito.

Para Nunes e cols. (2008), o irmão exerce um papel muito importante, pois a relação entre irmãos é uma das relações mais duradouras e a maneira com um irmão lida com o outro pode interferir no seu desenvolvimento. Esses autores analisam os efeitos positivos e negativos apresentados pelos irmãos diante da situação de ajudar nos cuidados de seus irmãos com NEE. Os efeitos positivos estão no desenvolvimento da maturidade, tolerância, interesse em carreiras humanitárias, senso de proximidade com a família e independência. Os efeitos negativos são a negligência parental, sobrecarga, ressentimentos e falta de atividades sociais. Os pais, diante da falta de suporte social, necessitam da ajuda dos filhos com desenvolvimento típico, que se sobrecarregam diante das responsabilidades com a família, deixando para segundo plano suas próprias atividades sociais. Devido à diferença diante da atenção dos pais, pode ocorrer uma rivalidade entre os irmãos, como evidenciam Silva e Fleith (2008).

Com relação às pesquisas sobre irmãos, a grande maioria é feita com díades de irmãos, sendo que, em muitas famílias, há mais de dois filhos (Nunes e cols., 2008). Ainda são escassas as pesquisas longitudinais para verificar como a família reage aos problemas enfrentados na adolescência e na fase adulta (Brito \& Dessen, 1999).
Apesar de o número de crianças incluídas na escola ter aumentado consideravelmente nos últimos anos, muitas crianças com NEE e seus familiares ainda sofrem preconceito (Brito \& Dessen, 1999; Falkenbach e cols., 2008; Souza \& Boemer, 2003). A nossa sociedade ainda está marcada pelo preconceito com a diferença, em que pessoas que estão "fora de um padrão" são excluídas, principalmente quando este padrão está relacionado ao nível de desenvolvimento cognitivo.

Diante dessa realidade, psicólogos e educadores devem trabalhar com a mudança nas concepções, provimento de informações (sobre desenvolvimento das crianças com deficiência, responsabilidade civil e direitos) dentro das escolas, comunidades e outros centros, e com a habilidade dos pais e das crianças para enfrentarem comportamentos preconceituosos da sociedade, de modo geral, e muitas vezes da família (Anache, 2005; Martinez, 2005), uma vez que muitos pais deixam os seus filhos em casa, sem ter contato com as pessoas e o público por temer uma reação negativa dos demais (Brito \& Dessen, 1999; Mendes e cols., 2004).

\section{Considerações finais}

É possível constatar, no campo da Psicologia, que há mais conhecimento diretamente relacionado às características das diversas formas com que se manifestam as NEE de crianças e pouco das pessoas diretamente envolvidas com os cuidados a elas destinados, seja de familiares ou outros cuidadores, ou mesmo de especialistas no assunto.

Tal revisão bibliográfica traz subsídios para que os profissionais da área da Saúde e da Educação conheçam as principais características das famílias de crianças com NEE (irmãos, pais, mães e demais membros) a fim de oportunizarem intervenções direcionadas às demandas destas crianças e, por consequência, favorecer o desenvolvimento infantil.

Dentre as pessoas envolvidas nos cuidados dos filhos com NEE, as mães ainda trazem mais informações consistentes, uma vez que, tradicionalmente, são as mais diretamente envolvidas no contato direto com tais crianças. Entretanto, os pais, aos poucos, começam a se envolver mais nesses casos e permitem a percepção do quanto tal envolvimento é importante e ajuda no progresso das relações familiares, resultando tanto em alívio para as mães, quanto em melhoria perceptível no desenvolvimento das crianças.

Quanto aos irmãos, nota-se, cada vez mais, o quanto eles podem se envolver e o que isso Ihes afeta na vida. Não parece, no geral, que apresentem grandes problemas em seu próprio desenvolvimento, mas muitos apresentam características afetadas em sua sociabilidade. Costumam se aproximar mais da família e adquirir certa precocidade em seu amadurecimento.

No geral, é possível perceber que os dados não permitem fazer generalizações conclusivas a respeito dos efeitos da presença de uma criança com NEE sobre as pessoas 
mais diretamente relacionadas a ela na família. Portanto, é importante investir em mais pesquisas (principalmente longitudinais), bem como na formação inicial e continuada de profissionais que trabalham diretamente com as pessoas com deficiências para produzir progressos específicos nesses tratamentos.

Como indicações de possíveis estudos e intervenções futuras, consideram-se: (a) revisão da literatura no âmbito da literatura internacional, já que foram encontrados poucos estudos da temática, no contexto brasileiro; (b) realização de estudos a respeito do papel do pai no desenvolvimento do filho com deficiência; (c) realização de mais pesquisas a respeito da relação dos irmãos, mais velhos e mais novos, junto ao irmão com deficiência; (d) mais estudos longitudinais, tendo em vista a fase adulta de pessoas com NEE e seus familiares; (e) programas de intervenção do psicólogo com os profissionais da saúde responsáveis por transmitir o diagnóstico e orientar a família quanto ao nascimento e ao desenvolvimento de crianças com deficiência.

Em relação à limitação do estudo, foi feita uma revisão com poucos artigos, mas que mostra a realidade de pesquisas na área no Brasil. Atualmente, vê-se um maior movimento no interesse de pesquisas e políticas públicas na área, mas que ainda é menor quando se compara a investimentos com outras populações.

\section{Referências}

Alencar, E. M. L. S. (2007). Características socioemocionais do superdotado: questões atuais. Psicologia em estudo 12(2), 371378

Anache, A. A. (2005). O psicólogo nas redes de serviços de Educação Especial: desafios em face da inclusão. Em A. M. Martinez (Org.), Psicologia escolar e compromisso social (pp. 115-134). Campinas, SP: Editora Átomo \& Alínea.

Araújo, E. A. C. (2004). Parceria família-profissional em educação especial: promovendo habilidades de comunicação efetiva. Em E. G. Mendes, M. A. Almeida \& L. C. A. Williams (Orgs.), Avanços recentes em Educação Especial (pp. 175-178). São Carlos: EDUFSCar.

Bee, H. (2008). A criança em desenvolvimento. Porto Alegre: Artmed.

Brasil. (2007). Política Nacional de Educação Especial na Perspectiva da Educação Inclusiva. Brasília: MEC/SEESP.

Brito, A. M. W., \& Dessen, M. A. (1999). Crianças surdas e suas famílias: um panorama geral. Psicologia: Reflexão e Crítica, 12(2), $1-11$.

Canho, P. G. M., Neme, C. M. B., \& Yamada, M. O. (2006). A vivência do pai no processo de reabilitação da criança com deficiência auditiva. Estudos de Psicologia, 23(3), 261-269.
Chacon, M. C. M. (2007). A deficiência mental e auditiva no olhar dos irmãos não deficientes. IV Congresso Brasileiro Multidisciplinar de Educação Especial, 1-11. Londrina, PR.

Cia, F., Williams, L., \& Aiello, A. L. R. (2005). Influências paternas no desenvolvimento infantil: revisão de literatura. Revista Psicologia Escolar e Educacional, 9(2), 225-233.

Cozby, P.C. (2006). Métodos de pesquisa em ciência do comportamento. São Paulo: Editora Atlas.

Dallabrida, A. M. (2007). As famílias e a classe especial em um colégio de elite. Revista Brasileira de Educação Especial, 13(3), 459-478.

Del Prette, Z. A. P., Martini, M. L. F. P., \& Del Prette, A. (2005) Contribuições do referencial das habilidades sociais para uma abordagem sistêmica na compreensão do processo de ensino aprendizagem. Interações, 10(20), 57 - 72.

Domingues, A.F., Motti, T.F.G. \& Palamin, M.E.G. (2008). O brincar e as habilidades sociais na interação da criança com deficiência auditiva e mãe ouvinte. Estudos de Psicologia, 25(1), 37-44.

Dessen, M. A., \& Silva, N. L. P. (2004). A família e os programas de intervenção: tendências atuais. Em E. G. Mendes, M. A. Almeida \& L. C. A. Williams (Orgs.), Avanços recentes em Educação Especial (pp.179-187). São Carlos, SP: EDUFSCar.

Dysson, L. L. (1997). Fathers and mothers of school-age children with developental disabilities: Parental stress, family functioning, and social support. American Journal on Mental Retardation, 102(3), 267-279

Erickson, M. F., \& Kurz-Reimer, K. (1999). Early intervention: Where we're been and where we're. Em M. F. Erickson \& K. Kurz-Reimer (Orgs.), Infants, toddlers, and families: A framework for support and intervention (pp.1-26). New York: Guilford Press.

Falkenbach, A. P, Drexsler, G., \& Werle, V. (2008). A relação mãe/ criança com deficiência: sentimentos e experiências. Ciência \& Saúde Coletiva, 13, 2065-2073.

Fávero, M. A. B., \& Santos, M. A. (2005). Autismo infantil e estresse familiar: uma revisão de literatura. Psicologia: Reflexão e Crítica, 18(3), 358-369.

Fiamenghi, G. A., \& Messa, A. A. (2007). Pais, filhos e deficiência: estudos sobre as relações familiares. Psicologia: Ciência e Profissão, 27(2), 236-245.

Filho, D. P., \& Santos, J. A. (2001). Metodologia científica. São Paulo: Editora Futura.

Floyd, F. J., \& Phillippe, K. A. (1993). Parental interactions with children with and without mental retardation: Behavior management, coerciveness, and positive exchange. American Jornal on Mental Retardation, 97(6), 673-684. 
Góes, F. A. B. (2006). Um encontro inesperado: os pais e seu filho com deficiência mental. Psicologia: Ciência e Profissão 26(3), 450461.

Gomes, V. F., \& Bosa, C. (2004). Estresse e relações familiares na perspectiva de indivíduos com Transtornos Globais do Desenvolvimento. Estudos de Psicologia 9(3), 553-561.

Gomide, P. I. C. (2003). Estilos parentais e comportamento antisocial. Em A. Del Prette \& Z.A.P. Del Prette (Orgs.), Habilidades sociais, desenvolvimento e aprendizagem (pp. 21-60). Campinas, SP: Alínea.

Guralnick, M. J. (1998). Effectiveness of early intervention for vulnerable children: A developmental perspective. American Journal of Mental Retardation, 102(4), 319-345.

Glidden, L. M., \& Floyd, F. J. (1997). Disaggregating parental depression and family stress in assessing families of children with developmental disabilities: A multisample analysis. American Journal on Mental Retardation, 102(3), 250-266.

Henn, C. G., Piccinini, C. A., \& Garcias, G. L. (2008). A família no contexto da Síndrome de Down: revisando a literatura. Psicologia em estudo, 13(3), 485-493.

Höher, S. P., \& Wagner, A. D. L. (2006). A transmissão do diagnóstico e de orientações a pais de crianças com necessidades especiais: a questão da formação profissional. Estudos de Psicologia, 23(2), 113-125.

Marchesi, A. (2004). Desenvolvimento e educação de crianças surdas. Em C. Coll, A. Marchesi \& J. Palácios (Orgs.). Desenvolvimento psicológico e educação: transtornos do desenvolvimento e necessidades educativas especiais (pp. 171-192). Porto Alegre: Artmed.

Martinez, A. M. (2005). Inclusão escolar: desafios para o psicólogo. Em A. M. Martinez (Orgs.), Psicologia escolar e compromisso social (pp. 95-114). Campinas, SP: Átomo \& Alínea.

Matsukura, T. S., Marturano, E. M., Oishi, J., \& Borasche, G. (2007). Estresse e suporte social em mães de crianças com necessidades especiais. Revista Brasileira de Educação Especial,13(3), 415428.

Mendes, E. G. (2006)A radicalização do debate sobre inclusão escolar no Brasil, Revista Brasileira de Educação,11(33), 387-405.

Mendes, E. G. M., Almeida, M. A., \& Williams, L. C. A. (2004). Temas em Educação Especial - avanços recentes. São Carlos, SP: EDUFSCar.

Mendes, E. G., Nunes, L. R. O. P., \& Ferreira, J. R.(2002). Diagnóstico e caracterização de indivíduos com necessidades educacionais especiais: produção científica nacional entre 1981 e 1998. Temas em Psicologia da SBP, 10(1), 11-26.
Mettrau, M. B., \& Reis, H. M. M. S. A. (2007). Políticas Públicas: altas habilidades/superdotação e a literatura especializada no contexto da educação especial/inclusiva. Ensaio: Avaliação de Políticas Públicas da Educação, 15(57), 489-510.

Negrin, N. S., \& Cristante, F. (1996). Resources and stress in parents with a mentally retarded child: A quantitative approach. Em M. Cusinato (Org.), Research on family: Resources and needs across the world (pp. 493-506). Milão: LED - Edicioni Universitarie.

Nunes, C. C., \& Aiello, A. L. R. (2008). Interação entre irmãos: deficiência mental, idade e apoio social da família. Psicologia: Reflexão e Crítica, 21(1), 42-50.

Nunes, C. C., Silva, N. C. B., \& Aiello, A. L. R. (2008). As contribuições do papel do pai e do irmão do indivíduo com necessidades especiais na visão sistêmica da família. Psicologia: Teoria e Pesquisa, 24(1), 37-44.

Palácios, J. (2004). Mudança e desenvolvimento durante a idade adulta e a velhice. Em C. Coll, A. Marchesi \& J. Palácios (Orgs.), Desenvolvimento psicológico e educação - Psicologia evolutiva (pp. 371-388). Porto Alegre: Artmed.

Paniagua, G. (2004). As famílias de crianças com necessidades educativas especiais. Em C. Coll, A. Marchesi \& J. Palácios (Orgs.), Desenvolvimento psicológico e educação: transtornos do desenvolvimento e necessidades educativas especiais (pp. 330346). Porto Alegre: Artmed.

Petean, E. B. L., \& Suguihura, A. L. M. (2005). Ter um irmão especial: convivendo com a Síndrome de Down. Revista Brasileira de Educação Especial, 11(3), 445-460.

Ribeiro, S. F. R., Yamada, M. O., \& Tavano, L. A. (2007). Vivência de mães de crianças com deficiência auditiva em sala de espera. Psicologia em Revista, 13(1), 91-106.

Schmidt, C., \& Bosa, C. (2003). A investigação do impacto do autismo na família: Revisão crítica da literatura e proposta de um novo modelo. Interação em Psicologia, 7(2), 111-120.

Silva, P. N. L. (2000). Crianças pré-escolares com síndrome de Down e suas interações familiares. Dissertação de mestrado, Universidade de Brasília, Brasília.

Silva, N. L. P., \& Dessen, M. A. (2001). Deficiência mental e família: implicações para o desenvolvimento da criança. Psicologia: Teoria e Pesquisa, 17(2), 133-141.

Silva, N. L. P., \& Dessen, M. A. (2002). Síndrome de Down: etiologia, caracterização e impacto na família. Interação em Psicologia, 6(2), 167-176.

Silva, N. L. P., \& Dessen, M. A. (2007). Crianças com e sem Síndrome de Down: valores e crenças de pais e professores. Revista Brasileira de Educação Especial, 13(3), 429-446. 
Silva, P. V. C., \& Fleith, D. S. (2008). A influência da família no desenvolvimento da superdotação. Revista Semestral da Associação Brasileira de Psicologia Escolar e Educacional, 12(2), 337-346.

Souza, L. G. A., \& Boemer, M. R. (2003). O ser - com o filho com deficiência mental - alguns desvelamentos. Paidéia, 13(26), 209219.

Tanaka, E. D. O., \& Manzini, E. J. (2005). O que os empregadores pensam sobre o trabalho da pessoa com deficiência?. Revista Brasileira de Educação Especial, 11(2), 273-294.
Williams, L. C. A., \& Aiello, A. L. R. (2004). O empoderamento de famílias: o que é e como medi-lo. Em E. G. Mendes, M. A. Almeida \& L. C. A. Williams (Orgs.), Avanços recentes em Educação Especial (pp.197-202). São Carlos, SP: EDUFSCar.

Yaegashi, S. F. R., Miranda, N. C., \& Komagrone, K. L. (2001). Alunos de classes especiais e sua família: algumas reflexões. Em M. C. Marquezine, M. A. Almeida \& E. D. O. Tanaka (Orgs.), Perspectivas multidisciplinares em Educação Especial II (pp.409-439). Londrina, PR: Editora UEL.

\section{Sobre as autoras}

\section{Paula Cruz Goitein}

Fabiana Cia (fabianacia@uniararas.br)

Universidade Federal de São Carlos

\section{Envio de correspondência:}

A/C de Fabiana Cia

Departamento de Psicologia

Rua Dr. Maximiliano Baruto, n. 500

CEP: 13607-339 - Araras - São Paulo

O presente trabalho é derivado do Trabalho de Conclusão de Curso da primeira autora, sob orientação da segunda autora. 